\title{
Influence of non-inherited maternal HLA-DR antigens on susceptibility to rheumatoid arthritis
}

I E van der Horst-Bruinsma, J M W Hazes, G M Th Schreuder, T R D J Radstake, P Barrera, L B A van de Putte, D Mustamu, D van Schaardenburg, F C Breedveld, R R P de Vries

Departments of Rheumatology

I E van der

Horst-Bruinsma

$\mathrm{J} M \mathrm{~W}$ Hazes

D Mustamu

F C Breedveld

and

Immunohaematology

and Bloodbank

G M Th Schreuder

R R P de Vries

Leiden University

Medical Centre, the

Netherlands

Jan van Breemen Institute Amsterdam, the Netherlands

D van Schaardenburg

University Hospital

Nijmegen, the

Netherlands

T R D J Radstake

P Barrera

L B A van de Putte

Correspondence to: Dr I E van der

Horst-Bruinsma, Leiden

University Medical Centre,

Department of

Rheumatology, C4-R, PO

Box 9600, 2300 RC Leiden,

the Netherlands.

Accepted for publication

9 September 1998

\begin{abstract}
Objective-It has recently been observed that non-inherited maternal DR4 antigens (NIMAs) of DR4 negative rheumatoid arthritis (RA) patients were increased compared with non-inherited paternal DR4 antigens (NIPAs). The aim of this study was to determine the prevalence of non-inherited DR4 antigens and DRB1 alleles in parents of RA patients.

Methods-HLA-DR serology and DRB1 typing was performed in $97 \mathrm{RA}$ patients and their parents. NIMA and NIPA frequencies were compared, stratified according to the presence of DR4 and/or the shared epitope (SE).

Results-In DR4 negative patients, NIMA DR4 was increased compared with NIPA DR4 (OR 3.10, 95\% CI 0.76, 12.70). When combined with results from a previous study this increase was significant (OR $3.65,95 \%$ CI $1.29,10.31)$. The NIMA effect of SE positive DR4 subtypes in this study (OR 4.73, 95\% CI 0.94, 23.8) was stronger than the NIMA effect of combined SE positive DRB1 alleles (OR 2.19 95\% CI $0.36,13.22$ ).

Conclusions-The association between non-inherited maternal HLA-DR4 alleles and the susceptibility to RA was observed in two independent populations.

(Ann Rheum Dis 1998;57:672-675)
\end{abstract}

The susceptibility to rheumatoid arthritis (RA) is associated with the presence of certain HLA DRB1 alleles sharing a common sequence of aminoacids at position $70-74$ of the DRbeta chain, which is known as the shared epitope (SE).$^{1-3}$ Particularly the $\mathrm{DRB} 1{ }^{\star} 0401$ and ${ }^{\star} 0404$ alleles are strongly associated with RA. ${ }^{2-7}$ We have previously proposed that not only inherited factors but also non-inherited HLA-antigens, especially those of the mother (NIMA), can influence the susceptibility to RA. Evidence for this hypothesis was obtained by the finding of an increased frequency of a DR4 NIMA of DR4 negative RA patients (RR 3.5) compared with DR4 non-inherited paternal antigens (NIPAs). ${ }^{8}$ In the DR4 positive patients the NIMA DR6 was increased (RR 3.2) and the NIMA DR3 was decreased. No evidence for such a NIMA effect was found in another study that mainly analysed DR4 positive patients from multicase families. ${ }^{9}$

The aim of this study was: (1) to test the NIMA hypothesis for the serologically defined DR4, DR3, and DR6 antigens in another population of RA patients. (2) To test the NIMA hypothesis for DRB1 alleles, taking into account the SE.

\section{Methods}

Ninety seven consecutive patients with RA fulfilling the 1987 ARA criteria ${ }^{10}$ were recruited in three outpatient clinics: 26 from Leiden (Leiden University Medical Centre), 48 from Amsterdam (Jan van Breemen Institute), and 23 from Nijmegen (University Hospital). The patients in Leiden were not included if they had participated in the former NIMA study. ${ }^{8}$ Both parents of all included patients had to be alive. All patients and their parents were interviewed to gain information about first degree relatives with RA. Blood samples were drawn from all patients and their parents to perform HLA class II typing.

\section{HLA TYPING}

Serological HLA-DR typing as well as generic DRB1 typing was performed with a polymerase chain reaction and biotin labelled sequence specific oligonucleotide (PCR-SSO) method as described previously. ${ }^{11}$ This method allows medium resolution DRB1 typing. DRB $1^{\star} 04$ subtyping (DRB1*0401-0411) was performed by group specific amplification of DNA from all DRB $1{ }^{\star} 04$ positive subjects and hybridisation with relevant SSOs. ${ }^{12}$ In this way it was possible to distinguish homozygosity and heterozygosity for all DRB $1^{\star} 04$ alleles. With the same technique the alleles known to express the shared epitope-that is, DRB $1^{\star} 0101,{ }^{\star} 0102,{ }^{\star} 0401$, ${ }^{\star} 0404,{ }^{\star} 0405,{ }^{\star} 0408,{ }^{\star} 0410$, and ${ }^{\star} 1001$ were identified. DRB $1^{\star} 06$ alleles were subtyped in $\star 1301 /{ }^{\star} 1302$, ${ }^{\star} 1303$, and ${ }^{\star} 1401$. Differentiation between ${ }^{\star} 1301$ and ${ }^{\star} 1302$ was not possible.

The HLA DRB1 typings of 421 healthy organ donors served as controls for the HLA profile of the RA patients.

NIMA or NIPA were defined as serological DR antigens or DRB1 alleles present in respectively mother or father and not inherited by the patient.

Table 1 Comparison of frequencies of rheumatoid arthritis (RA) associated DRB1 alleles between $R A$ patients and controls

\begin{tabular}{llll}
\hline$H L A$ & $\begin{array}{l}\text { Patients } \\
n=97(\%)\end{array}$ & $\begin{array}{l}\text { Controls } \\
n=421(\%)\end{array}$ & OR $(95 \%$ CI $)$ \\
\hline DR4 & $58(60)$ & $95(23)$ & $5.10(3.20,8.13)$ \\
SE & $72(74)$ & $178(42)$ & $3.93(2.40,6.45)$ \\
${ }^{*} 0401 / \star_{0404}$ & $56(58)$ & $94(22)$ & $4.75(2.99,7.55)$ \\
\hline
\end{tabular}

SE: shared epitope, that is, DRB $1{ }^{\star} 0101,{ }^{\star} 0102,{ }^{\star} 0401,{ }^{\star} 0404$, ${ }^{\star} 0405,{ }^{\star} 0408$, and ${ }^{\star} 1001$ alleles. ${ }^{\star} 0401 /{ }^{\star} 0404$ : DRB $1{ }^{\star} 0401$ or DRB1 ${ }^{\star} 0404$ allele present. 
Table 2 Frequency of HLA-DR non-inherited maternal(NIMA) and paternal antigens (NIPA) in RA patients

\begin{tabular}{llll}
\hline \multicolumn{4}{l}{ DR4 negative patients $(n=39)$} \\
\hline$H L A$ & NIMA & NIPA & OR $(95 \%$ CI) \\
\hline DR3 & 6 & 7 & $0.83(0.25,2.74)$ \\
DR4 & 8 & 3 & $3.10(0.76,12.70)$ \\
DR6 & 6 & 11 & $0.46(0.15,1.41)$ \\
DR4 positive patients $(n=58)$ & 11 & $0.49(0.17,1.44)$ \\
DR3 & 6 & 9 & $1.13(0.42,3.04)$ \\
DR4 & 10 & 11 & $0.68(0.25,1.85)$ \\
DR6 & 8 & &
\end{tabular}

To compare the NIMA and NIPA frequencies in non-RA families HLA-DR typing data from the healthy Dutch families of the previous study were used. ${ }^{8}$

STATISTICS

The association between the presence and absence of HLA-DR3, DR4, and DR6 as a NIMA or a NIPA were calculated using odds ratios with $95 \%$ confidence intervals. This analysis was done in patients stratified according to the presence or absence of DR4. The frequencies of DRB $1{ }^{\star} 0401$ and/or ${ }^{\star} 0404$ or all SE positive alleles as NIMAs or NIPAs were calculated separately in the same way after stratification for the presence or absence of these DRB1 alleles or the SE. The data of the previous study ${ }^{8}$ were reanalysed calculating the odds ratio and confidence intervals instead of the relative risk. The odds ratio of the DR4 positive NIMA compared with DR4 positive NIPA in DR4 negative patients was calculated after combining the results of the previous study with this study.

\section{Results}

Most patients were women (85\%) and, because of the selection for both parents alive, were young with a median age of RA onset at 29 years (range 16-53). The median age of disease onset did not differ among DR4 negative compared with DR4 positive patients (29 v 28 years, Kruskall-Wallis (KW) test $\mathrm{p}=0.51$ ) or in $\mathrm{DRB} 1{ }^{\star} 0401 /{ }^{\star} 0404$ negative versus DRB $1{ }^{\star} 0401 /{ }^{\star} 0404$ positive patients $(29 \approx 27$ years, $\mathrm{KW} \mathrm{p}=0.21$ ) or in $\mathrm{SE}$ negative versus $\mathrm{SE}$ positive patients (29 $v 26$ years, KW p=0.29).

The frequencies of the DR4, SE, and the SE positive $\mathrm{DRB} 1{ }^{\star} 04$ alleles ${ }^{\star} 0401 /{ }^{\star} 0404$ alleles were increased in patients compared with healthy blood donors (table 1). The association of DR4 and DRB1*04 subtypes with RA did not differ much because most $\mathrm{DRB}^{\star}{ }^{\star} 04$ subtypes were $\mathrm{DRB} 1{ }^{\star} 0401$ and ${ }^{\star} 0404$ alleles.

Table 3 Frequency of HLA-DR non-inherited maternal (NIMA) and paternal antigens (NIPA) in DR4 negative $R A$ patients from this study and the study of Ten Wolde ${ }^{8}$ and in DR4 negative non-RA controls

\begin{tabular}{lllll}
\hline & & NIMA & NIPA & OR $(95 \%$ CI $)$ \\
\hline This study & DR4+ & 8 & 3 & $3.10(0.76,12.70)$ \\
Ten Wolde et al & DR4- & 31 & 36 & \\
& DR4+ & 12 & 2 & $4.26(0.88,20.62)$ \\
& DR4- & 38 & 27 & \\
Both studies & DR4+ & 20 & 5 & $3.65(1.29,10.31)$ \\
& DR4- & 69 & 63 & \\
Controls & DR4+ & 8 & 9 & $0.87(0.31,2.42)$ \\
& DR4- & 58 & 57 & \\
\hline
\end{tabular}

Table 4 Frequencies of $R A$ associated DR4 subtypes $\left({ }^{\star} 0401\right.$ and/or $\left.{ }^{\star} 0404\right)$ as NIMAs and NIPAs of ${ }^{\star} 0401$ and/or ${ }^{\star} 0404$ negative $R A$ patients and ${ }^{\star} 0401$ and/or *0404 positive $R A$ patients

\begin{tabular}{|c|c|c|c|c|}
\hline & \multicolumn{2}{|c|}{$\begin{array}{l}{ }^{*} 0401 /{ }^{*} 0404- \\
\text { patients }(n=41)\end{array}$} & \multicolumn{2}{|c|}{$\begin{array}{l}{ }^{*} 0401 /{ }^{*} 0404+ \\
\text { patients }(n=56)\end{array}$} \\
\hline & NIMA & NIPA & NIMA & NIPA \\
\hline$\star_{0} 0401$ & 4 & 1 & 7 & 6 \\
\hline$\star_{0} 0404$ & 4 & 1 & 2 & 1 \\
\hline Total & 8 & 2 & & 7 \\
\hline OR $(95 \% \mathrm{CI})$ & \multicolumn{2}{|c|}{$4.73(0.94,23.82)$} & \multicolumn{2}{|c|}{$1.34(0.46,3.89)$} \\
\hline
\end{tabular}
both absent $(-)$.

Firstly, we analysed the NIMA and NIPA frequencies at serological level using the same methodology as in the previous study. ${ }^{8}$ In the 39 DR4 negative patients, the DR4 positive NIMAs were increased compared with the DR4 positive NIPAs, but this increase failed to reach significance (OR 3.10, 95\% CI 0.76, 12.70, table 2). Neither an increase of DR6NIMA nor a strong decrease of DR3-NIMA was found in DR4 positive patients (table 2).

The results of the former study ${ }^{8}$ were reanalysed in the same way as in this study calculating the odds ratio instead of the relative risk. Thus it appeared that the increase of DR4 NIMAs compared with DR4 NIPAs of DR4 negative RA patients was also non-significant (OR 4.26, 95\% CI 0.88, 20.62) (table 3). When the results of this study are combined with those of the previous study the increase of DR4 NIMAs of DR4 negative RA patients was significant (OR 3.65, 95\% CI 1.29, 10.31, table 3).

In non-RA control families ${ }^{8}$ the frequency of DR4 positive NIMAs was equal to that of DR4 positive NIPAs in DR4 negative controls (table 3).

Next we focused on the DRB $1^{\star} 04$ alleles as defined by PCR-SSO typing. In the DR4 negative patients the eight DR4 NIMAs were: DRB $1{ }^{\star} 0401$ alleles in four cases and the ${ }^{\star} 0404$ also in four cases; the three DR4 NIPAs were: $\mathrm{DRB} 1{ }^{\star} 0401,{ }^{\star} 0402$, and ${ }^{\star} 0404$. In the DR4 positive patients the 10 DR4 NIMAs were: $\mathrm{DRB} 1{ }^{\star} 0401$ in seven cases, ${ }^{\star} 0403$ in one case, and ${ }^{\star} 0404$ in two cases, whereas the nine DR4 NIPAs were: ${ }^{\star} 0401$ in six cases, the ${ }^{\star} 0404$ in one, and the ${ }^{\star} 0407$ in two cases. The DRB $1{ }^{\star} 0405$ and ${ }^{\star} 0408$ alleles were not found in this population. The other DRB $1{ }^{\star} 04$ alleles carrying the SE motif $\left({ }^{\star} 0401\right.$ and $\left.{ }^{\star} 0404\right)$ were increased as NIMAs compared with the DRB $1{ }^{\star} 0401$ and/or ${ }^{\star} 0404$ positive NIPAs in patients lacking these alleles (OR 4.73, 95\% CI $0.94,23.82$, table 4).

The effect of all SE positive alleles as a NIMA compared with the SE positive NIPA (OR 2.19, 95\% CI 0.36, 13.22, table 5) was smaller than the effect of the SE positive DR4 subtypes (table 4 ).

To exclude a bias caused by other genetic factors that might play a part in multicase families, the family history of the patients was obtained. Thirteen patients belonged to multicase families: five (19\%) from Leiden, four $(8 \%)$ from Amsterdam, and four $(17 \%)$ from Nijmegen respectively. A separate analysis of 
Table 5 Frequency of shared epitope (SE) alleles as non-inherited maternal (NIMA) and paternal antigens (NIPA) in SE positive and SE negative RA patients

\begin{tabular}{|c|c|c|c|c|}
\hline \multirow[b]{2}{*}{$S E$} & \multicolumn{2}{|c|}{$S E$-patients $(n=25)$} & \multicolumn{2}{|c|}{ SE+patients $(n=72)$} \\
\hline & $N I M A$ & NIPA & $N I M A$ & NIPA \\
\hline$\star_{0} 0101$ & & 1 & 5 & 12 \\
\hline${ }^{\star} 0102$ & & & 1 & \\
\hline$\star_{0} 0401$ & \multicolumn{2}{|l|}{1} & 10 & 7 \\
\hline$\star^{\star} 0404$ & \multicolumn{2}{|l|}{2} & 4 & 2 \\
\hline$\star 1001$ & 1 & 1 & 2 & \\
\hline Total & 4 & 2 & 22 & 21 \\
\hline OR $(95 \% \mathrm{CI})$ & \multicolumn{2}{|c|}{$2.19(0.36,13.22)$} & \multicolumn{2}{|c|}{ OR $1.07(0.52,2.18)$} \\
\hline
\end{tabular}

the NIMA frequencies in these patients was not possible because of the small number of DR4 negative patients ( 3 of 13 ).

\section{Discussion}

The increase of DR4 NIMAs compared with DR4 NIPAs of DR4 negative RA patients found in this study was in accordance with the previous study ${ }^{8}$ and does reach significance if the results of both studies are combined (OR $3.65,95 \%$ CI 1.29, 10.31).

Focusing on the DR4 subtypes that were significantly associated with RA in the patients studied, the frequency of $\mathrm{DRB} 1^{\star} 0401$ and/or ${ }^{\star} 0404$ NIMAs of ${ }^{\star} 0401$ and/or ${ }^{\star} 0404$ negative RA patients was increased compared with the NIPAs carrying these alleles. This increase (OR 4.73, 95\% CI 0.94, 23.82) was stronger than that observed for DR4 (OR 3.10, 95\% CI $0.76,12.70)$. The effect of the SE positive NIMA of SE negative patients was less impressive (OR 2.19, 95\% CI 0.36, 13.22).

Therefore we conclude that the risk of RA seems to be increased in patients who are genetically not predisposed (that is, DR4 and shared epitope negative) but have contact during their fetal period with $\mathrm{DRB} 1^{\star} 0401$ and/or ${ }^{\star} 0404$ positive HLA antigens derived from the mother. Because we ascertained RA patients with a young age of onset (median age 29 years) as both parents had to be alive for patients to be included in the study, this conclusion may only be valid for RA with early onset.

To our knowledge there has been only one other study on the subject of the NIMA hypothesis and the onset of RA. ${ }^{9}$ This study showed no increase of either maternal DR4 or the HLA-DRB1 SE compared with the same non-inherited paternal antigens. These RA patients were all derived from multicase families, who might have a different genetic background than sporadic RA patients, the latter group constituting the majority of the RA patients. Of more importance is that there were too few DR4 negative patients in that study to allow an analysis of NIMAs of DR4 negative patients. As the DR4 NIMA effect was observed only in DR4 negative patients, the results of that study are not in contrast with our findings.

In two other HLA associated autoimmune diseases, insulin dependent diabetes mellitus and myasthenia gravis, no increase of noninherited maternal disease predisposing HLA antigens was found. ${ }^{13} 14$

The mechanism of the influence of the noninherited maternal HLA antigens might be that these HLA antigens are associated with the acquisition or course of a viral infection in the mother, which then could be transmitted to the fetus. However, we favour the hypothesis that these non-inherited maternal HLA antigens themselves are transmitted to the fetus and exert their effect through the shaping of the $\mathrm{T}$ cell repertoire of the child in utero. ${ }^{15}$ The observation that $50 \%$ of the patients that receive multiple blood transfusions do not form antibodies against the non-inherited maternal HLA antigens supports this hypothesis. ${ }^{16}$ Moreover several studies show that maternal cells do enter the fetal circulation $^{17-20}$ and thus might change the immune system of the child.

The finding that DR4-subtypes that carry the SE motif (DRB $1^{\star} 0401$ and $\left.{ }^{\star} 0404\right)$ seem to have a stronger NIMA effect than DR4, is compatible with a pathogenetic influence of these alleles on RA. During the fetal period ${ }^{\star} 0401$ and/or ${ }^{\star} 0404$ positive $T$ cells, molecules or peptides of the mother might change the $\mathrm{T}$ cell repertoire of the child, which might make it prone to develop RA later in life.

In conclusion, in $\mathrm{DRB} 1{ }^{\star} 0401$ and ${ }^{\star} 0404$ negative RA patients the frequency of $\mathrm{DRB} 1{ }^{\star} 0401$ or $\mathrm{DRB} 1{ }^{\star} 0404$ alleles is increased in the mothers compared with the fathers. This suggests that maternal ${ }^{\star} 0401$ and/or ${ }^{\star} 0404$ positive cells, molecules or peptides may exert a predisposing effect on the development of RA in fetuses who are negative for these alleles.

We are very grateful to P D M de Buck and P W van Schendel, who participated in the preparation of this research project and collected many patients, W Verduyn who supervised the DRB1 subtyping and R v d Wijngaard who took part in collecting the
patients.

We would also like to thank J J van Rood for his support in all the stages of the study.

1 Nepom GT, Nepom BS. Prediction of susceptibility to rheumatoid arthritis by human leukocyte antigen genotyping. Rheum Dis Clin North Am 1992;18:785-92.

2 Nelson JL, Dugowson CE, Koepsell TD, Voigt LF, Nelson JL, Dugowson CE, Koepsell TD, Voigt LF,
Branchaud AM, Barrington RA, et al. Rheumatoid factor, HLA-DR4, and allelic variants of DRB1 in women with recent-onset rheumatoid artritis. Arthritis Rheum 1994;37: 673-80.

3 Gough A, Faint J, Salmon M, Hassell A, Wordsworth P, Pilling D, et al. Genetic typing of patients with inflammatory arthritis at presentation can be used to predict outcome. Arthritis Rheum 1994;37:1166-70.

4 Deighton CM, Cavanagh G, Rigby AS, Lloyd HL, Walker DJ. Both inherited HLA-haplotypes are important in the predisposition to rheumatoid arthritis. $\mathrm{Br}$ J Rheumatol 1993;32:893-8.

5 Salmon M, Wordsworth P, Emery P, Tunn E, Bacon PA, Bell JI. The association of HLA DR $B$ alleles with self-limiting and persistent forms of early symmetrical polyarthritis. Br J Rheumatol 1993;32:628-30.

6 Wordsworth BP, Stedeford J, Rosenberg WM, Bell JI. LimWordsworth BP, Stedeford J, Rosenberg WM, Bell JI. Lim-
ited heterogeneity of the HLA class II contribution to susited heterogeneity of the HLA class II contribution to susceptibility to rheumatoid arthritis is suggested by positive
associations with HLA-DR4, DR1 and DRw10. Br J Rheumatol 1991;30:178-80.

matol 1991;30:178-80.
7 MacGregor A, Ollier W, Thomson W, Jawaheer D, Silman A. HLA-DRB $1^{\star} 0401 / 0404$ genotype and rheumatoid arthritis: increased association in men, young age at onset, and disease severity. J Rheumatol 1995;22:1032-6.

8 Wolde ten S, Breedveld FC, Vries de RRP, D'Amaro J, Rubenstein P, Schreuder GMT, et al. Influence of non-inherited maternal HLA antigens on occurence of rheumatoid arthritis. Lancet 1993;341:200-2.

9 Silman AJ, Hay EM, Worthington J, Thomson W, Pepper L, Davidson J, et al. Lack of influence of non-inherited maternal HLA-DR alleles on susceptibility to rheumatoid arthritis. Ann Rheum Dis 1995;54:311-3.

10 Arnett FC, Edworthy SM, Bloch DA, McShane DJ, Fries $\mathrm{JF}$, Cooper NS, et al. The American Rheumatism Association 1987 revised criteria for the classification of rheumation 1987 revised criteria for the classification of
toid arthritis. Arthritis Rheum 1988;31:315-24.

11 Verduyn W, Doxiadis IIN, Anholts J, Drabbels JJM, Naipal A, D'Amaro J, et al. Biotinylated DRB sequence-specific 
oligonucleotides; comparison to serologic HLA-DR typing of organ donors in Eurotransplant. Hum Immunol

12 Gao X, Olsen NJ, Pincus T, Stastny P. HLA-DR alleles with naturally occurring amino acid substitutions and risk for development of rheumatoid arthritis. Arthritis Rheum 1990;33:939-46.

13 Kockum I, Wassmuth R, Holmberg E, Michelsen B, Lenmark A. Inheritance of MHC class II genes in IDDM studied in population-based affected and control families. Diabetologia 1994;37:1105-12.

14 Matsuki K, Maeda H, Nomura Y, Segawa M. Influence of non-inherited maternal HLA on disease development. Lancet 1993;341:639-40.

15 Zhang L, Rood JJ van, Claas FHJ. The T-cell repertoire is not dictated by self antigens alone. Res Immunol 1991;142:441-5.
16 Claas FHJ, Gijbels Y, Velden-de Munck J van der, Rood JJ van. Induction of B cell unresponsiveness to inherited maternal HLA antigens during fetal life. Science 1988;241: $1815-17$

7 Lo YMD, LO ESF, Watson N, Noakes L, Sargent IL, Thilaganathan $\mathrm{B}$, et al. Two-way cell traffic between mother and fetus: biologic and clinical implications. Blood 1996;88:4390-5.

8 Hall JM, Lingenfelter P, Adams SL, Lasser D, Hansen JA, Bean MA. Detection of maternal cells in human umbilical cord blood using fluorescence in situ hybridization. Blood 1995;86:2829-32.

19 Artlett CM, Smith B, Jimenez SA. Identification of fetal DNA and cells in skin lesions from women with systemic sclerosis. N Engl J Med 1998;338:1186-91.

20 Nelson JL. Microchimerism and autoimmune disease. N Engl J Med 1998;338:1224-5. 